\title{
Multiple-contact Thermoelastic Oscillations
}

\author{
P. D. Howell ${ }^{\mathrm{a}}$, J. R. Barber ${ }^{\mathrm{b}}$ \& J. R. Ockendon ${ }^{\mathrm{a}}$ \\ a Mathematical Institute, Andrew Wiles Building, Oxford OX2 6GG, UK; \\ ${ }^{b}$ Department of Mechanical Engineering, University of Michigan, 2350 Hayward \\ Street, Ann Arbor, MI 48109-2125, USA
}

June 8, 2018

\begin{abstract}
This paper derives and analyses a model for the thermoelastic response of a system of pins sliding relative to a rigid rough plane. The model not only describes the configurations observed in the experiments of Barber [1] but also reveals the possibility of other modes, depending on the relative importance of wear, thermal expansion and thermal conduction.
\end{abstract}

Keywords: Thermoelastic instability; mathematical modelling; stability analysis; dynamical systems. 



\title{
Multiple-contact Thermoelastic Oscillations
}

\author{
June 8, 2018
}

\begin{abstract}
This paper derives and analyses a model for the thermoelastic response of a system of pins sliding relative to a rigid rough plane. The model not only describes the configurations observed in the experiments of Barber [1] but also reveals the possibility of other modes, depending on the relative importance of wear, thermal expansion and thermal conduction.
\end{abstract}

Keywords: Thermoelastic instability; mathematical modelling; stability analysis; dynamical systems.

\section{Introduction}

When two bodies slide against each other, heat is generated at the interface due to friction, and the resulting temperature distribution causes thermoelastic distortion which affects the contact pressure distribution. This coupled thermo-mechanical process can be unstable, in which case it is generally referred to as 'frictionally-excited thermoelastic instability' or 'TEI' [1] 3]. In the absence of wear, the system is asymptotic to a steady state in which only a part of the original contact area remains in contact [4], and locally high temperatures can be generated, known as hot spots. The same frictional interactions cause material removal (wear) at the interface at a rate which is generally assumed to be proportional to the rate of frictional energy dissipation [5]. This tends to have a stabilising effect on the process [6], but in many cases the wear rate is too slow to prevent the instability. However, in this case a steady state cannot be sustained. Eventually the hot spots will be 
worn away and the load must be transferred to another part of the nominal contact surface.

This process was demonstrated experimentally in [1] for the sliding contact of two extended bodies, and also for an idealized system in which three pins mounted on one flat plate are brought into contact with a second flat plate that moves parallel to the first one. In an earlier paper [7] a mathematical model was developed for one- and two-pin configurations. This model reveals that wear always prevails over thermal expansion in the one-pin case and that instantaneous load transfer cannot occur in the two-pin case. However, load transfer can occur on time scales much shorter than the periods of single pin contact. These predictions are confirmed by numerical simulations of the two-pin model in [8] and by asymptotic analysis of the two-pin model in $[9]$.

Our ultimate aim in this paper is to show that, in the three-pin situation considered in [1], it is likely that the configurations can similarly oscillate between relatively long periods of one-pin contact and shorter intervals of simultaneous contact in which load transfer occurs. Our analysis will reveal that many different kinds of oscillatory responses are also possible and this will lead us to conjecture that the complexity will increase as the number of pins is further increased. Hopefully a model with many pins will provide an explanation for the hot spots that have been observed in sliding contact between rough surfaces (see [1]).

The paper begins with a simple model that couples the mechanisms of heat conduction, wear and thermoelasticity in the pins. We will show that, by using a complementarity formulation, this model can be made almost as concise for $N$ pins as it is for two pins. Moreover, for the two-pin problem, it is possible to further reduce the model to a scalar first-order ordinary integro-differential equation from which reliable numerical solutions can be obtained. Next, we will consider the many kinds of instability that can 
occur for a uniform state in which all the pins have the same length and are in contact under the same force. Finally, we will show that the three-pin problem can be reduced to a two-dimensional integro-differential equation for which numerical solutions can also be easily obtained.

\section{The $N$-pin model}

\section{Governing equations and boundary conditions}

We consider $N$ metal pins, each with one end clamped to a metal arm held at ambient temperature $T_{\mathrm{amb}}$. The temperature $T_{j}$ in $\operatorname{pin} j=1, \cdots, N$ satisfies the heat equation

$$
\kappa \frac{\partial^{2} T_{j}}{\partial X^{2}}=\frac{\partial T_{j}}{\partial t}
$$

where $\kappa$ is the thermal diffusivity (assumed constant), $X$ is the material (i.e. Lagrangian) distance from the clamped base, and $t$ is time. As we will see below, the thermoelastic deformation of each pin is small, so that any metric coefficients arising from expressing the spatial derivatives in the Lagrangian frame may be neglected. At the clamped end, we impose Newton's law of cooling, that is,

$$
k \frac{\partial T_{j}}{\partial X}=h_{\mathrm{A}}\left(T_{j}-T_{\mathrm{amb}}\right) \quad \text { at } X=0
$$

where $k$ is the thermal conductivity and $h_{\mathrm{A}}$ is a heat transfer coefficient.

The other end of each pin is pressed with force $F_{j}$ against a wheel moving tangentially at constant speed $V$. The resulting heat generation, at a rate proportional to $F_{j} V$, is modelled by the boundary condition

$$
k \frac{\partial T_{j}}{\partial X}=\mu h_{\mathrm{W}} V F_{j} \quad \text { at } X=L_{j}
$$

where $\mu$ is the coefficient of friction, and $h_{\mathrm{W}}$ is a second heat transfer coeffi-

cient. The material length $L_{j}(t)$ of pin $j$ decreases over time due to frictional 
wear at a rate proportional to $F_{j} V$, assuming Archard's Law, and hence

$$
\frac{\mathrm{d} L_{j}}{\mathrm{~d} t}=-\beta \mu V F_{j}
$$

where $\beta$ is a material constant of proportionality. Neglecting the effects of elastic waves, the physical (Eulerian) length $\ell_{j}$ is recovered from $L_{j}$ using

$$
\ell_{j}=L_{j}\left(1-\frac{F_{j}}{E A}\right)+\frac{\alpha_{\mathrm{T}}}{3} \int_{0}^{L_{j}} T_{j} \mathrm{~d} X
$$

where $E, A$ and $\alpha_{\mathrm{T}}$ are the Youngs modulus, cross-sectional area and thermal expansion coefficient of each pin. The terms on the right-hand side of equation (5) respectively represent the effects of elastic compression, by the imposed force $F_{j}$, and thermal expansion.

Finally, we impose a constant net force $F$, while noting that it is impossible for the wheel to exert a tensile force on any pin. In addition, the compressive force $F_{j}$ is nonzero only if pin $j$ makes contact with the wheel, and therefore we can write the complementarity conditions

$$
\begin{gathered}
F_{j} \geq 0, \quad\left(M-\ell_{j}\right) F_{j}=0 \quad(j=1, \cdots, N) \\
\text { and } \quad \sum_{j} F_{j}=F
\end{gathered}
$$

where $M=\max _{j}\left(\ell_{j}\right)$ is the length of the longest pin.

\section{Dimensionless model}

We non-dimensionalise as in [7], defining

$$
\begin{array}{ll}
\left(X, L_{j}, \ell_{j}\right)=L_{0}\left(\tilde{X}, \tilde{L}_{j}, \tilde{\ell}_{j}\right), & t=\frac{L_{0}^{2}}{\kappa} \tilde{t}, \\
T_{j}=T_{\mathrm{amb}}+\frac{h_{\mathrm{W}} \mu V F L_{0}}{k} \tilde{T}_{j}, & F_{j}=F \tilde{F}_{j}
\end{array}
$$


where $L_{0}$ is a typical initial length for each pin. With tildes dropped, the dimensionless version of the model (1)-(6) reads

$$
\begin{array}{cc}
\frac{\partial^{2} T_{j}}{\partial X^{2}}=\frac{\partial T_{j}}{\partial t} & 0<X<L_{j}(t) \\
\frac{\partial T_{j}}{\partial X}=h T_{j} & X=0 \\
\frac{\partial T_{j}}{\partial X}=F_{j} & X=L_{j} \\
\frac{\mathrm{d} L_{j}}{\mathrm{~d} t}=-\delta \eta F_{j}, & \ell_{j}=L_{j}\left(1-\delta F_{j}\right)+\delta \alpha \int_{0}^{L_{j}} T_{j} \mathrm{~d} X
\end{array}
$$

for $j=1, \cdots, N$, where

$$
\begin{gathered}
F_{j} \geq 0, \quad M-\ell_{j} \geq 0, \quad\left(M-\ell_{j}\right) F_{j}=0 \quad(j=1, \cdots, N) \\
\sum_{j} F_{j}=1
\end{gathered}
$$

The four dimensionless parameters

$$
\delta=\frac{F}{E A}, \quad \alpha=\frac{E A \alpha_{\mathrm{T}}}{3} \frac{h_{\mathrm{W}} \mu V L_{0}}{k}, \quad \eta=\frac{\beta \mu V L_{0} E A}{\kappa}, \quad h=\frac{h_{\mathrm{A}} L_{0}}{k}
$$

respectively measure the typical elastic strain, thermal expansion, wear rate, and heat transfer with the arm. For the experiments described in [1], the applied force $F$ results in such a small relative compression of a pin that $\delta$ is expected to be extremely small. We take the distinguished asymptotic limit in which $\delta \rightarrow 0$ while the other three parameters are $O(1)$, so that all the relevant physical effects are retained in the model at lowest order.

We infer from equation (11) that both $L_{j}$ and $\ell_{j}$ remain close to their initial values, which we take to be 1 by our normalisation. Therefore we can write

$$
L_{j}=1-\delta D_{j}, \quad \ell_{j}=1+\delta d_{j}
$$


where $D_{j}$ measures the accumulated wear of pin $j$, while $d_{j}$ measures the net lengthening of pin $j$, resulting from a competition between thermal expansion, elastic compression, and wear.

To leading order in $\delta$, the thermal problem (8)-10 reduces to

$$
\begin{array}{cl}
\frac{\partial^{2} T_{j}}{\partial X^{2}}=\frac{\partial T_{j}}{\partial t} & 0<X<1 \\
\frac{\partial T_{j}}{\partial X}=h T_{j} & X=0 \\
\frac{\partial T_{j}}{\partial X}=F_{j} & X=1
\end{array}
$$

Substituting equation (14) into equation (11) and letting $\delta \rightarrow 0$, we find that $D_{j}$ and $d_{j}$ are governed by the equations

$$
\frac{\mathrm{d} D_{j}}{\mathrm{~d} t}=\eta F_{j}, \quad D_{j}=\alpha \int_{0}^{1} T_{j} \mathrm{~d} X-F_{j}-d_{j}
$$

We eliminate $D_{j}$ from equation (18) to obtain

$$
\frac{\mathrm{d}}{\mathrm{d} t}\left(F_{j}+d_{j}\right)=(\alpha-\eta) F_{j}-\left.\alpha \frac{\partial T_{j}}{\partial X}\right|_{X=0}
$$

The problem is closed by the rescaled version of equation (12), namely

$$
\begin{gathered}
F_{j} \geq 0, \quad m-d_{j} \geq 0, \quad\left(m-d_{j}\right) F_{j}=0 \quad(j=1, \cdots, N) \\
\sum_{j} F_{j}=1
\end{gathered}
$$

Given suitable initial conditions for $T_{j}(X, 0), F_{j}(0)$ and $d_{j}(0)$, equations (15)(17), (19) and (20) in principle determine $T_{j}(X, t), F_{j}(t)$ and $d_{j}(t)$ for $t>0$.

Since the dimensionless forces $F_{j}$ must sum to unity, if we sum the problem (15)-17) over $j$, we see that the sum of the temperatures satisfies constant boundary conditions and must, therefore, converge to a timeindependent solution. Ignoring an irrelevant initial transient phase, we deduce that the sums of the dependent variables are determined by

$$
\sum_{j} T_{j}(X, t)=X+\frac{1}{h}, \quad \sum_{j} d_{j}(t)=-\eta t, \quad \sum_{j} F_{j}(t)=1
$$


In principle, the conservation identities (21) allow us to reduce the dimension of the problem from $N$ to $N-1$; we will see how this can be done in practice when $N=2$ and $N=3$ below.

\section{Green's function formulation}

By expressing the solution of the PDE problem (15)-(17) in terms of a Green's function, we can transform equation (19) into the integro-differential equation

$$
\frac{\mathrm{d}}{\mathrm{d} t}\left(F_{j}+d_{j}\right)=(\alpha-\eta) F_{j}-\alpha Q\left[F_{j}\right]
$$

where

$$
Q[F](t)=\int_{0}^{t} F(\tau) G(t-\tau) \mathrm{d} \tau
$$

For the problem (15)-(17), for example by taking a Laplace transform, we can explicitly solve for the Green's function (which depends parametrically on the coefficient $h$ ) in the form

$$
G(h ; t)=\frac{1}{2 \pi \mathrm{i}} \int_{\epsilon-\mathrm{i} \infty}^{\epsilon+\mathrm{i} \infty} K(h, \sqrt{p}) \mathrm{e}^{p t} \mathrm{~d} p
$$

where $\epsilon>0$ and, for future reference, we have introduced the function

$$
K(h, s):=\frac{1}{\cosh s+(s / h) \sinh s}
$$

By summing over the poles in $K$, we can alternatively express $G$ as the infinite series

$$
G(h ; t)=\sum_{n=0}^{\infty} \frac{2 h^{2} \cos \left(k_{n}(h)\right)}{h+\sin ^{2}\left(k_{n}(h)\right)} \mathrm{e}^{-k_{n}(h)^{2} t}
$$

where $k_{n}(h)$ is the root in the range $n \pi<k<(n+1 / 2) \pi$ of the transcendental equation

$$
k \tan k=h
$$


In the limit $h \rightarrow \infty$ we have $k_{n}(h) \rightarrow(n+1 / 2) \pi$, and hence equation 26 may be simplified somewhat to

$$
G(\infty ; t)=2 \pi \sum_{n=0}^{\infty}(-1)^{n}\left(n+\frac{1}{2}\right) \mathrm{e}^{-(n+1 / 2)^{2} \pi^{2} t}
$$

The Green's function $G(h ; t)$ is plotted versus $t$ for various values of $h$ in figure 1. We also show as black dotted and black dashed curves, respectively, the small- $t$ and large- $t$ asymptotic limits, namely

$$
\begin{aligned}
G(h ; t) & \sim \frac{2 h}{\sqrt{\pi t}} \mathrm{e}^{-1 / 4 t} & & \text { as } t \rightarrow 0 \\
G(\infty ; t) & \sim \frac{1}{\sqrt{\pi} t^{3 / 2}} \mathrm{e}^{-1 / 4 t} & & \text { as } t \rightarrow 0 \\
G(h ; t) & \sim \frac{2 h^{2} \cos \left(k_{0}(h)\right)}{h+\sin ^{2}\left(k_{0}(h)\right)} \mathrm{e}^{-k_{0}(h)^{2} t} & & \text { as } t \rightarrow \infty \\
G(\infty ; t) & \sim \pi \mathrm{e}^{-\pi^{2} t / 4} & & \text { as } t \rightarrow \infty
\end{aligned}
$$

The Green's function allows us to express the evolution equation equation (22) as a stand-alone integro-differential equation and thus eliminate the need to solve for the temperature. This approach is also more general: different models for the heat flow (for example the possibility of heat loss out of the sides of the pins) could in principle be incorporated by modifying the Green's function appropriately. Finally, we note that useful insights into the behaviour of the dynamical system (20) and (22) might be obtained by substituting equation 26 for a simpler model Green's function.

We now consider the solution of 22 subject to the complementarity conditions 20 in the case $N=2$.

\section{The 2-pin problem}

When $N=2$, we may simplify the problem by defining

$$
d_{1}(t)-d_{2}(t)=U(t), \quad F_{1}(t)=\frac{1+\Lambda(t)}{2}, \quad F_{2}(t)=\frac{1-\Lambda(t)}{2}
$$


where $\Lambda(t)=F_{1}(t)-F_{2}(t) \in[-1,1]$, then the complementarity condition (20) becomes

$$
\left(1-\Lambda^{2}\right) U=0, \quad(1+\Lambda) U \geq 0, \quad(1-\Lambda) U \leq 0, \quad\left(1-\Lambda^{2}\right) \geq 0
$$

while the wear equation $(19)$ implies that

$$
\frac{\mathrm{d}}{\mathrm{d} t}(\Lambda+U)-(\alpha-\eta) \Lambda+\alpha Q[\Lambda]=0
$$

To impose the complementarity condition (34), it is helpful to introduce the quantity

$$
H=\Lambda+U
$$

Then equation (34) defines $\Lambda$ as a continuous function of $H$, namely

$$
\Lambda(H)=\frac{1}{2}(|H+1|-|H-1|)
$$

as plotted in figure 2, Equation (35) may then be expressed in the form

$$
\frac{\mathrm{d} H}{\mathrm{~d} t}-(\alpha-\eta) \Lambda(H)+\alpha Q[\Lambda(H)]=0
$$

Given a suitable initial condition for $H(0)$, equation (38) provides a closed problem for $H(t)$, with the function $\Lambda(H)$ defined by equation (37).

In figure 3 we show numerical solutions of the 2-pin problem (38) obtained with $h=2$ and fixed initial condition $H(0)=0.2$. In figures $3(\mathrm{a})$ and $3(\mathrm{~b})$ we show the results of setting two different values of $\eta$ and then varying the value of $\alpha$. In either case, we observe four possible behaviours. For the smallest values of $\alpha$, the solution for $\Lambda$ decays exponentially to zero, corresponding to a uniform equilibrium state, having both pins in contact with equal lengths and temperatures. As $\alpha$ increases through some critical value, the uniform state loses stability, and instead the system undergoes nonlinear oscillations, with the two pins taking turns to support the applied load. For larger values of $\alpha$, the oscillations become increasingly violent. 
In the next section, we will explain the change in stability observed in figure 3 by performing a linear stability analysis on equation (38). We will also briefly consider some implications for the N-pin problem.

\section{Equilibrium and stability analysis}

\section{The 2-pin problem}

Equation (38) admits the trivial steady solution $H=0$, in which both pins are in contact with equal contact forces and equal temperature profiles. Small perturbations about this base state satisfy a linearised problem where the function $\Lambda(H)$ in equation (38) is replaced by $H$. Indeed, this linearisation is exact for $|H| \leq 1$, and therefore perturbations grow, oscillate or decay exponentially until one of the pins loses contact. To find the growth rate, we seek solutions of the form

$$
H(t)=A \mathrm{e}^{\sigma t}
$$

By substituting into the linearised evolution equation (38), we find that the exponential growth rate $\sigma$ satisfies the transcendental equation

$$
\sigma+\eta-\alpha+\alpha K(h, \sqrt{\sigma})=0
$$

where $K$ is again the function defined by equation (25).

\section{The $N$-pin problem}

Before analysing the equation (40) for the linear growth rate $\sigma$, we first show how the linear theory generalises to the general case of $N$ pins. Again there is an equilibrium state in which all the pins are in contact with equal forces, lengths and temperatures, i.e. the problem (20), (22), admits the solution

$$
F_{j}=\frac{1}{N}, \quad d_{j}=-\frac{\eta t}{N} \quad \text { for } j=1, \cdots, N
$$


We linearise about the base state equation (41) by adding infinitesimal perturbations of the form $\tilde{F}_{j} \mathrm{e}^{\sigma t}$ and $\tilde{d}_{j} \mathrm{e}^{\sigma t}$ to $F_{j}$ and $d_{j}$ respectively. This procedure results in a system of $N$ problems of the form

$$
\begin{aligned}
(\sigma+\eta-\alpha) \tilde{F}_{j}+\sigma \tilde{d}_{j} & =-\alpha \tilde{F}_{j} K(h, \sqrt{\sigma}) & & (j=1, \cdots, N) \\
\tilde{d}_{j} & =\tilde{m} & & (j=1, \cdots, N) \\
\sum_{j} \tilde{F}_{j} & =0 & &
\end{aligned}
$$

Note in particular that the length perturbations $\tilde{d}_{j}$ must all be equal, and equation (42) may therefore be rearranged to

$$
[\sigma+\eta-\alpha+\alpha K(h, \sqrt{\sigma})] \tilde{F}_{j}=-\sigma \tilde{m} \quad(j, 1, \cdots, N)
$$

where $K$ is again the function defined by equation (25). Now applying equation (44), we see that nontrivial solutions can exist only if $\tilde{m}=0$. Thus the linear growth rate $\sigma$ for the general $N$-pin problem satisfies exactly the same transcendental equation equation (40) as for the 2-pin problem. Furthermore, the eigenspace of linear perturbations is highly degenerate, consisting of all solutions of the form $\tilde{F}_{j} \mathrm{e}^{\sigma t}$ subject only to the condition equation 44t that all the amplitudes $\tilde{F}_{j}$ sum to zero.

Next we will attempt to divide up the parameter space into regions where the system exhibits different qualitative behaviour, depending on the real and imaginary parts of the dominant growth rate, i.e. the $\operatorname{root}(\mathrm{s}) \sigma$ of equation (40) with the largest real part. Special attention will be paid to the dependence of the stability on $\alpha$, which measures the influence of thermal expansion. 


\section{Analysis of parameter space}

\section{Zero growth rate}

First we note from equation (40) that $\sigma$ approaches zero as either $\eta$ or $h$ approaches zero, with

$$
\sigma \sim \frac{\eta h}{\alpha+h(\alpha / 2-1)} \quad \text { as } \eta \rightarrow 0, \quad \sigma \sim \frac{\eta h}{\alpha-\eta} \quad \text { as } h \rightarrow 0
$$

Therefore, as $\eta \rightarrow 0$ we see that $\sigma$ is real and positive for $\alpha>2 h /(h+2)$ and negative for $\alpha<2 h /(h+2)$. As $h \rightarrow 0$, we similarly have that $\sigma$ is positive for $\alpha>\eta$ and negative for $\alpha<\eta$.

\section{Pure imaginary growth rate}

Next we seek parameter values such that $\sigma$ is complex and its real part passes through zero by making the substitution

$$
\sigma= \pm 2 \mathrm{i} w^{2}
$$

with $w>0$ without loss of generality. By then taking real and imaginary parts of equation (40), we find that values of $\alpha$ and $\eta$ for which $\operatorname{Re}(\sigma)=0$ are given parametrically by

$$
\begin{aligned}
& \alpha=\frac{w^{2}\left[\left(h^{2}+2 w^{2}\right) \cosh 2 w+\left(h^{2}-2 w^{2}\right) \cos 2 w+2 h w(\sinh 2 w-\sin 2 w)\right]}{h \sinh w \sin w(h+w \operatorname{coth} w+w \cot w)}, \\
& \eta=\alpha-\frac{2 w^{2} \operatorname{coth} w \cot w[h+w \tanh w-w \tan w]}{h+w \operatorname{coth} w+w \cot w} .
\end{aligned}
$$

For a given value of $h$ and $w \in(0, \infty)$, equation 48 generates a multiply branched curve in the $(\alpha, \eta)$-plane. The relevant lowest branch where the dominant growth rate has zero real part corresponds to values of $w$ between 0 and $w_{*}(h)$, where $w_{*}(h)$ is the root between $\pi / 2$ and $\pi$ of the transcendental equation

$$
w_{*} \operatorname{coth} w_{*}+w_{*} \cot w_{*}=-h
$$


The resulting curve in the $(\alpha, \eta)$ plane with $h=2$ is plotted as a green curve in figure 4. As $w \rightarrow 0$, the parametrisation (48) approaches the critical point $(\alpha, \eta)=(2 h /(h+2), 0)$ identified above. At the other extreme where $w \rightarrow w_{*}(h)$, both $\alpha$ and $\eta$ tend to infinity, with

$$
\frac{\eta}{\alpha} \rightarrow \zeta_{*}(h):=1+\frac{2 h \sin w_{*}(h) \sinh w_{*}(h)}{w_{*}(h)\left(\sin 2 w_{*}(h)+\sinh 2 w_{*}(h)\right)}
$$

As shown by the green curve in figure 5 , this limiting gradient varies only very slightly, between 1 as $h \rightarrow 0$ and $1+\operatorname{sech} \pi \approx 1.086$ as $h \rightarrow \infty$.

\section{Stability exchange}

A double root $\sigma$ of equation (40) corresponds to a point where two real roots coalesce and turn into a complex conjugate pair, or vice versa. Such points therefore delineate regions of parameter space where the dominant growth rate is real or complex, i.e. where the temporal behaviour is monotonic or oscillatory.

At a positive double root, we let $\sigma=z^{2}$ in equation (40), where $z>0$ without loss of generality. Then the simultaneous equations

$$
z^{2}+\eta-\alpha+\alpha K(h, z)=2 z+\alpha \frac{\partial K(h, z)}{\partial z}=0
$$

provide two expressions for $\alpha$ and $\eta$, namely

$$
\begin{aligned}
& \alpha=\frac{2 z(h \cosh z+z \sinh z)^{2}}{h((h+1) \sinh z+z \cosh z)} \\
& \eta=\alpha-\frac{z\left(\left(2 h+z^{2}\right) \cosh z+(h+3) z \sinh z\right)}{(h+1) \sinh z+z \cosh z}
\end{aligned}
$$

For a given value of $h$ and $z \in(0, \infty)$, equation (52) parametrises a curve in the $(\alpha, \eta)$-plane across which the dominant growth rate switches from positive real to complex. The resulting curve with $h=2$ is plotted in orange in figure 4. As $z \rightarrow 0$, we see that $(\alpha, \eta)$ again approaches the critical point 
$(2 h /(h+2), 0)$. On the other hand, letting $z \rightarrow \infty$ in equation $(52)$ we see that

$$
\eta \sim \alpha-\log ^{2}(\alpha)+\cdots \quad \text { as } \alpha \rightarrow \infty
$$

Therefore $\eta / \alpha \rightarrow 1$ as $\alpha, \eta \rightarrow \infty$, regardless of the value of $h$, as illustrated by the orange line in figure 5 .

Next we perform an analogous calculation for negative double roots by letting $\sigma=-z^{2}$ in equation (40), resulting in the parametric representation

$$
\begin{aligned}
\alpha & =\frac{2 z(h \cos z-z \sin z)^{2}}{h((h+1) \sin z+z \cos z)}, \\
\eta & =\alpha+\frac{z\left(\left(z^{2}-2 h\right) \cos z+(h+3) z \sin z\right)}{(h+1) \sin z+z \cos z} .
\end{aligned}
$$

For fixed $h$ and $z \in(0, \infty)$, equation (54) parameterises a family of curves in the $(\alpha, \eta)$-plane, of which the lowest branch is the relevant one corresponding to the dominant growth rate. We therefore consider values of $z \in\left(0, z_{*}(h)\right)$, where $z_{*} \in(\pi / 2, \pi)$ satisfies the transcendental equation

$$
\frac{\tan z_{*}}{z_{*}}=-\frac{1}{h+1}
$$

The resulting curve in the $(\alpha, \eta)$-plane with $h=2$ is plotted in blue in figure 4. As $z \rightarrow 0$ we again approach the critical point $(\alpha, \eta)=(2 h /(h+$ $2), 0)$. At $z=z_{c}(h) \in(0, \pi / 2)$, satisfying the equation

$$
z_{c} \tan z_{c}=h
$$

there is a turning point corresponding to $(\alpha, \eta)=\left(0, z_{c}(h)^{2}\right)$. Finally, as $z \rightarrow z_{*}(h)$, both $\alpha$ and $\eta$ tend to infinity, with limiting gradient

$$
\frac{\eta}{\alpha} \rightarrow 1+\frac{2 h \sin z_{*}(h)}{2 z_{*}+\sin 2 z_{*}(h)}
$$

As shown by the blue curve in figure 5 , this value increases from 1 towards 2 as $h$ increases from 0 towards infinity. 


\section{Parameter space}

Now assembling all the ingredients, we see that the $(\alpha, \eta)$ parameter space for a fixed value of $h=2$ is as shown in figure 4 . The three marginal curves identified above divide the plane into four separate regions in which the dominant growth rate is either (1) real and negative, (2) complex with negative real part, (3) complex with positive real part, or (4) real and positive. The three curves all meet at the critical point $(\alpha, \eta)=(2 h /(h+2), 0)$, but otherwise do not intersect, as is confirmed by the ordering of the far-field gradients plotted in figure 5 .

This structure is similar to that for other nonzero values of $h$. As $h \rightarrow 0$, all three curves collapse onto the line $\eta=\alpha$, implying that the behaviour switches directly from monotonic decay to monotonic growth as $\alpha$ increases past $\eta$ in this singular limit. The planar cross-sections similar to figure 4 may thus be assembled into the three-dimensional parameter space illustrated in figure 6, in which the three surfaces delineate values of $(\alpha, \eta, h)$ corresponding to the four canonical behaviours enumerated above.

The computations shown in figure 3 confirm the parametric dependence predicted by the linear theory. For a fixed value of $\eta$ and increasing $\alpha$, we pass successively through the regimes corresponding to monotonic decay, oscillatory decay, oscillatory growth and monotonic growth. In the former two cases, the system ends up at the uniform equilibrium state, having both pins in contact with equal lengths and temperatures. In the latter two cases, the system ultimately undergoes violent oscillations, with each pin in turn taking the load and rapid load transfer between the two pins. The two sets of simulations shown in figures $3(\mathrm{a})$ and $3(\mathrm{~b})$ demonstrate that the same four characteristic behaviours occur both below and above the turning point at $(\alpha, \eta)=\left(0, z_{c}^{2}\right)$.

In the next section, we will consider the complications resulting from the introduction of a third pin. 


\section{The 3-pin problem}

\section{Problem formulation}

Now we show how the 3-pin problem may be formulated using a more general representation than that used above for the 2-pin problem. Since the forces $F_{j}$ must sum to unity, we can again reduce the dimensionality of the problem by defining the force differences

$$
\Lambda_{1}=F_{1}-F_{2}, \quad \Lambda_{2}=F_{2}-F_{3}, \quad \Lambda_{3}=F_{3}-F_{1}
$$

The vector $\boldsymbol{\Lambda}=\left(\Lambda_{1}, \Lambda_{2}, \Lambda_{3}\right)$ lives on the plane $\Lambda_{1}+\Lambda_{2}+\Lambda_{3}=0$, and the constraints (20) imply that $\boldsymbol{\Lambda}$ must lie on an equilateral triangle with vertices at $\boldsymbol{\Lambda}=(-1,1,0),(0,-1,1)$ and $(1,0,-1)$. States with all three pins in contact correspond to points on the interior of the triangle; states with two pins in contact correspond to the edges of the triangle and states with just one pin in contact to the vertices.

We continue to follow the recipe from the 2-pin problem by defining

$$
U_{1}=d_{1}-d_{2}, \quad U_{2}=d_{2}-d_{3}, \quad U_{3}=d_{3}-d_{1}
$$

and

$$
H_{j}=\Lambda_{j}+U_{j} \quad \text { for } j=1,2,3
$$

The vector $\boldsymbol{H}=\left(H_{1}, H_{2}, H_{3}\right)$ also lives on the plane $H_{1}+H_{2}+H_{3}=0$, which is sketched in figure 7(a). Our aim is to express $\boldsymbol{\Lambda}$ as a function of $\boldsymbol{H}$ for all points on this plane.

First we note that, for points on the interior of the equilateral triangle identified above, all of the forces $F_{j}$ are nonzero. It follows that $d_{j}=m$ and hence $U_{j}=0$ for all $m$, and therefore $\boldsymbol{\Lambda}=\boldsymbol{H}$ for all such points, as indicated

inside the triangle shown in figure $7(\mathrm{a})$.

Next consider an edge of the triangle, for example the one where pin 1 is out of contact, so that $F_{1}=0$ and hence $d_{1}<d_{2}=d_{3}$. For any point on the 
corresponding edge, we have

$$
\begin{aligned}
& \Lambda_{1}=-F_{2} \quad \Lambda_{2}=2 F_{2}-1 \quad \Lambda_{3}=1-F_{2} \\
& =H_{1}+U_{3} \quad=H_{2} \quad=H_{3}-U_{3}
\end{aligned}
$$

where $U_{3}>0$. By eliminating $F_{2}$, we obtain the solution

$$
\Lambda_{1}=-\frac{1}{2}\left(1+H_{2}\right), \quad \Lambda_{2}=H_{2}, \quad \Lambda_{3}=\frac{1}{2}\left(1-H_{2}\right)
$$

for $\boldsymbol{\Lambda}$. Furthermore, since $\boldsymbol{\Lambda}$ is constant when $H_{2}$ is constant with $H_{3}>\Lambda_{3}$, the solution (62) holds on lines pointing out of the triangle perpendicular to the edge $H_{3}-H_{1}=1$, as shown in figure 7(a), It is easy to see (or to argue by symmetry) that the same result holds for the other two edges of the triangle: the interior solution $\boldsymbol{\Lambda}=\boldsymbol{H}$ is just projected along outward perpendicular lines from each edge.

Finally, consider a vertex at which just one pin is in contact, pin 1 for example. Here we have $F_{1}=1$ and $F_{2}=F_{3}=0$ and hence

$$
\begin{aligned}
& \begin{array}{lll}
\Lambda_{1}=1 & \Lambda_{2}=0 & \Lambda_{3}=-1
\end{array} \\
& =H_{1}-U_{1} \quad=H_{2}+U_{1}+U_{3} \quad=H_{3}-U_{3}
\end{aligned}
$$

where $U_{3}<0<U_{1}$. Therefore we have $\boldsymbol{\Lambda}=(1,0,-1)$ in the sector $H_{3}+1<$ $0<H_{1}-1$ through the vertex $\boldsymbol{H}=(1,0,-1)$. Similar constant solutions hold in sectors through the other vertices, and thus complete the piecewise definition of $\boldsymbol{\Lambda}$ as a function of $\boldsymbol{H}$, as shown in figure 7(a).

We can extract from figure $7(\mathrm{a})$ definitions of each component of $\boldsymbol{\Lambda}$; for example, $\Lambda_{1}$ may be expressed in the form

$$
\begin{array}{r}
\Lambda_{1}(\boldsymbol{H})=-\frac{1}{2}\left(\left|H_{1}-1\right|-\left|H_{1}+1\right|\right) \mathcal{H}\left(-H_{1}+H_{2}+1\right) \mathcal{H}\left(-H_{3}+H_{1}+1\right) \\
+\frac{1}{4}\left(\left|H_{2}-1\right|-\left|H_{2}+1\right|-2\right) \mathcal{H}\left(H_{3}-H_{1}-1\right) \\
\quad \frac{1}{4}\left(\left|H_{3}-1\right|-\left|H_{3}+1\right|+2\right) \mathcal{H}\left(H_{1}-H_{2}-1\right)
\end{array}
$$


where $\mathcal{H}$ denotes the Heaviside function. The resulting piecewise linear function is plotted versus $\boldsymbol{H}$ in figure 7(b), Analogous expressions for the other components of $\Lambda$ may be found by permuting the indices in equation (64).

The evolution equation (22) may now be expressed in the form

$$
\frac{\mathrm{d} \boldsymbol{H}}{\mathrm{d} t}=(\alpha-\eta) \boldsymbol{\Lambda}(\boldsymbol{H})-\alpha Q[\boldsymbol{\Lambda}(\boldsymbol{H})]
$$

with $\boldsymbol{\Lambda}(\boldsymbol{H})$ determined as described above. In principle, the constraint $H_{1}+$ $H_{2}+H_{3} \equiv 0$ is preserved by equation (65), but in practice we parametrise the plane with

$$
H_{1}=-\frac{\sqrt{3}}{2} H_{x}-\frac{1}{2} H_{y}, \quad H_{2}=\frac{\sqrt{3}}{2} H_{x}-\frac{1}{2} H_{y}, \quad H_{3}=H_{y}
$$

so that equation 65 becomes a two-dimensional system for $\left(H_{x}, H_{y}\right) \in \mathbb{R}^{2}$.

\section{Numerical simulations}

In figures 8 and 9 , we show results of simulations of the 3-pin problem 65) with $h=2, \eta=2.5$ and $\alpha \in\{0.2,2.5,4.0,10.0\}$; these are the same parameters as used for the 2-pin problem in figure $3(\mathrm{~b})$,

Figures $8(\mathrm{a})$ and $8(\mathrm{c})$ show results with the smallest value $\alpha=0.2$. In figure 8(a) we plot the computed forces $\left(F_{1}, F_{2}, F_{3}\right)$ versus $t$, using initial conditions $\left(H_{x}, H_{y}\right)=(-3,3)$ at $t=0$, such that only pin 3 is in contact and $F_{1}=F_{2}=0$ at $t=0$. We observe that first pin 1 and then pin 2 come into contact, before all three forces decay monotonically to the stable equilibrium state $\left(F_{1}, F_{2}, F_{3}\right)=(1 / 3,1 / 3,1 / 3)$, as predicted by linear stability analysis. The same solution is plotted in the $\boldsymbol{H}$-plane in figure 8(c). We recall that the central equilateral triangle corresponds to points where all three pins are in contact; the numbers in the diagram indicate regions of phase space where each of the pins is in contact, delineated the dashed lines. We see clearly how the system evolves from pin 3, to pins 1 and 3, to all three pins being in contact, before finally converging to the stable node at the origin. 
The same initial conditions are used for the simulation with $\alpha=2.5$, whose results are shown in figures $8(\mathrm{~b})$ and $8(\mathrm{~d})$. Here, after all three pins come into contact, there is a brief period where pin 3 loses contact before finally the system converges to the stable spiral at $\boldsymbol{H}=\mathbf{0}, F_{1}=F_{2}=F_{3}=$ $1 / 3$, again in agreement with linear theory. The behaviours of the surface temperatures and pin lengths likewise display transient oscillations before settling down to the uniform equilibrium state.

In figure 9 we show numerical solutions of the 3-pin problem with larger values of $\alpha$ where the equilibrium solution is predicted to be unstable. Here we choose initial conditions $\left(H_{x}, H_{y}\right)=(0.2,0.2)$ at $t=0$, such that all three pins are initially in contact, and we can observe the growth of the instability.

Let us consider first figures $9(\mathrm{~b})$ and $9(\mathrm{~d})$, with the largest value of $\alpha=10$, so the large exponential growth rate causes the system to diverge rapidly. The system ends up in a periodic state where just one pin is in contact almost all of the time, with very abrupt transitions as the three pins take turns to support the load; the behaviour is qualitatively similar to the corresponding 2-pin solution shown in figure $3(\mathrm{~b})$. Figure $9(\mathrm{~d})$ helps to understand why this behaviour occurs: when the amplitude of the solution for $\boldsymbol{H}$ is large, most of the solution trajectory lies in the sectors in which just one pin is in contact, which take up the majority of the phase space.

Finally consider the solution plotted in figures 9(a) and 9(c) for the intermediate value $\alpha=4$.0. Here the equilibrium $\boldsymbol{H}=\mathbf{0}$ is predicted to be an unstable spiral, and indeed we observe oscillatory initial growth. However, it appears that growth rate in this case is too feeble to launch the system into a large-amplitude orbit as in figure $9(\mathrm{~d})$. Instead it gets stuck in an intermediate state characterised by apparently irregular periods of each pin being in and out of contact, and indeed the dynamics plotted in the $\boldsymbol{H}$-plane figure 9(c) gives every appearance of being chaotic.

In the original experiments of [1], the temperature at the surface of each 
pin and the separation distance between the block assembly and the wheel surface were recorded. For comparison with those results, we also plot in figure 10 the evolutions of $T_{j}(1, t)$ and $d_{j}(t)$ with $t$ for $j=1,2,3$, recovered from the above simulation with $\alpha=10$. The black dashed curve in figure 10(b) shows the maximum pin displacement, which corresponds to the net displacement of the block assembly relative to the wheel surface. The observed behaviours of the pin temperatures and the net displacement agree qualitatively with the experimental results shown in figure 4 of [1]. As each pin in turn comes into contact with the wheel, its temperature rises, and the consequent thermal expansion causes an increase in the pin length. Eventually, the length starts to decrease again as wear becomes increasingly important, and ultimately the pin loses contact, the temperature rapidly drops, and the cycle begins again with a different pin.

\section{Discussion}

In this paper, we have derived a mathematical model for the thermomechanical experiments carried out by Barber [1], in which the temperatures, forces and lengths of a system of metal pins are coupled through the effects of frictional heating, thermal expansion and wear. The complicated switch in behaviour that occurs whenever each pin gains or loses contact with the moving plate is captured by a set of simultaneous complementarity conditions (20). The result is a dynamical system in which the coupling is both non-smooth and non-local, in that each pin is coupled to every other pin.

We have focused on the cases where the number $N$ of pins is either 2 or 3. In either case, we showed that the problem can be projected onto a solution space of dimension $N-1$ by formulating it in terms of differences between the temperatures, forces and lengths of successive pins. This approach, which permits the problem to be solved numerically with relative 
ease and also allows the results to be neatly visualised, can in principle also be used for higher values of $N$. With $N=4$, for example, we would end up with a dynamical system equivalent to (65), with the dependent variable $\boldsymbol{H}(t)$ a vector in $\mathbb{R}^{3}$, a central tetrahedron corresponding to simultaneous contact of all 4 pins, and the extrapolations of the vertices, edges and faces corresponding to regions where 1, 2 and 3 pins are in contact, respectively.

Our mathematical model always admits an equilibrium solution in which all of the pins have equal temperatures, forces and lengths. We used a linear stability analysis to identify the regions of parameter space where this uniform state is unstable. The linear analysis also reveals a highly degenerate solution space, in which all admissible linear modes grow or decay at precisely the same rate, reflecting the fact that all of the pins are indistinguishable from each other. It would be interesting to incorporate spatial coupling between neighbouring pins, for example through elastic deformation of the base plate, which would result in a non-trivial eigenvalue problem in place of (45), and thus to a distinguished set of linear modes reflecting the heterogeneity in the inter-pin coupling.

In situations where the uniform state is unstable, the system inevitably evolves into a nonlinear oscillatory solution. For the case $N=2$, we have found that the ultimate state is always periodic, with contact being repeatedly exchanged between the two pins at regular intervals. When $N>2$, the possibilities are more exotic: in particular, with $N=3$ we find that the system can produce apparently chaotic oscillations in some parameter regimes, and one can imagine the range of behaviours that might be observed if the number of pins were increased further.

Aside from our linear stability analysis, we have not attempted an exhaustive parameter sweep to determine precisely when each type of behaviour occurs. Indeed, it is not clear to what extent the ultimate solution that the system adopts is determined solely by the parameter values. The analysis 
carried out in [9] shows that it is possible for the uniform state and a nonlinear periodic solution to be simultaneously stable. In such a situation, the system may converge to either of the available stable solutions depending on the initial conditions, and there is also the possibility of hysteretic switching between them.

Our results reveal that the parameter $\alpha$, which measures the relative importance of thermal expansion, plays a key role. If $\alpha$ is small, then the uniform state in which all the pins are in contact is stable. However, when $\alpha$ is large, the uniform state is unstable, and instead the system undergoes oscillations, whose amplitude and violence increases with increasing $\alpha$. This behaviour is analysed in detail for the case $N=2$ in 9 by performing a formal asymptotic analysis in the limit $\alpha \rightarrow \infty$. For the case $N=3$, the simulations shown in figures $9(\mathrm{~b})$ and $9(\mathrm{~d})$ illustrate how, when $\alpha$ is large, the phase space is largely filled by regions where just one pin is in contact, and the same would be true for higher values of $N$. This observation explains why simultaneous contact between pins is only possible for short times, and the load is repeatedly born by a single pin: it is gratifying that this prediction is in qualitative agreement with the experimental results of [1].

\section{References}

[1] J. R. Barber, "Thermoelastic instabilities in the sliding of conforming solids," Proc. R. Soc. Lond. A, vol. 312, no. 1510, pp. 381-394, 1969.

[2] T. A. Dow and R. A. Burton, "Thermoelastic instability of sliding contact in the absence of wear," Wear, vol. 19, no. 3, pp. 315-328, 1972.

[3] J. R. Barber, Contact Mechanics. Dordrecht: Springer, 2018. 
[4] R. A. Burton, S. R. Kilaparti, and V. Nerlikar, "A limiting stationary configuration with partially contacting surfaces," Wear, vol. 24, no. 2, pp. 199-206, 1973.

[5] J. F. Archard, "Contact and rubbing of flat surfaces," J. Appl. Phys., vol. 24 , no. 8, pp. 981-988, 1953.

[6] T. A. Dow and R. A. Burton, "The role of wear in the initiation of thermoelastic instabilities of rubbing contact," J. Lubrication Tech., vol. 95, no. 1, pp. 71-75, 1973.

[7] J. R. Ockendon and J. R. Barber, "A model for thermoelastic contact oscillations," IMA J. Appl. Math., vol. 81, no. 4, pp. 679-687, 2016.

[8] A. Akande, "A model for thermoelastic contact oscillations," Master's thesis, University of Oxford, UK, 2016.

[9] P. D. Howell, "Analysis of a dynamical system arising in thermoelastic contact," submitted to SIAM J Appl Math., 2018. 


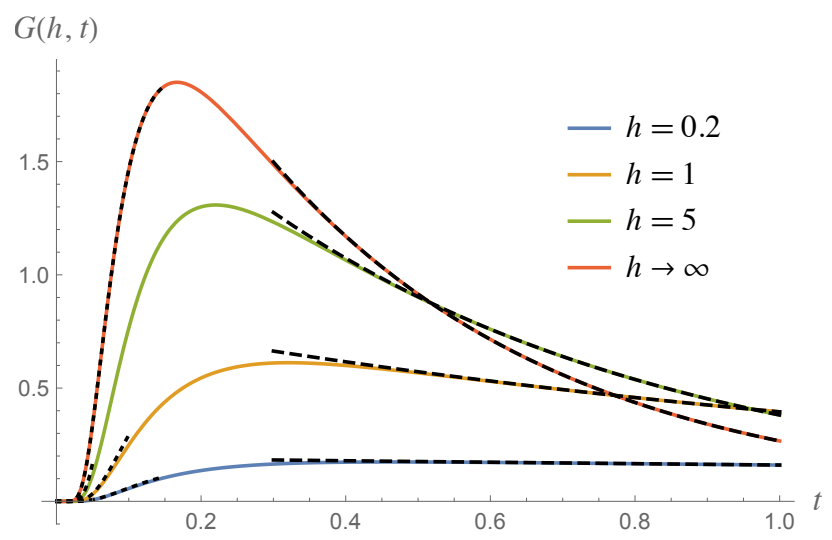

Figure 1: The Green's function defined by equation (26) plotted versus $t$ for different values of $h$; the limiting case as $h \rightarrow \infty$ is given by equation (28). The small- $t$ and large- $t$ asymptotic limits, given by equations $(29)-(32)$, are plotted as black dotted and black dashed curves, respectively. 


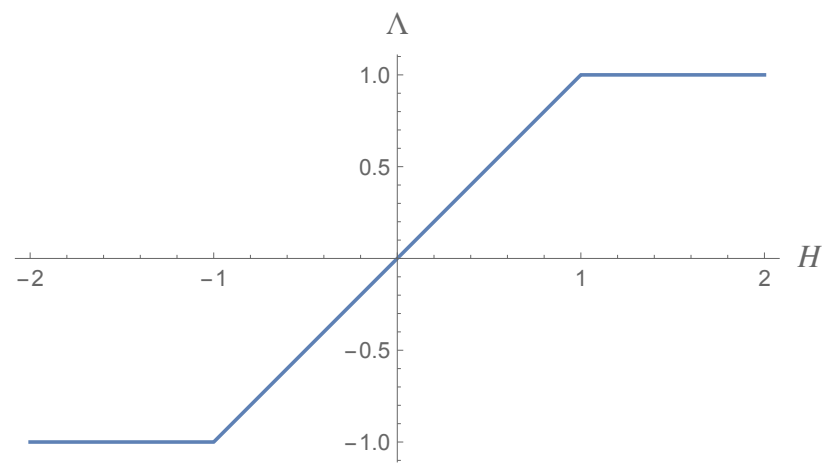

Figure 2: The function $\Lambda(H)$ defined by equation $(37)$. 


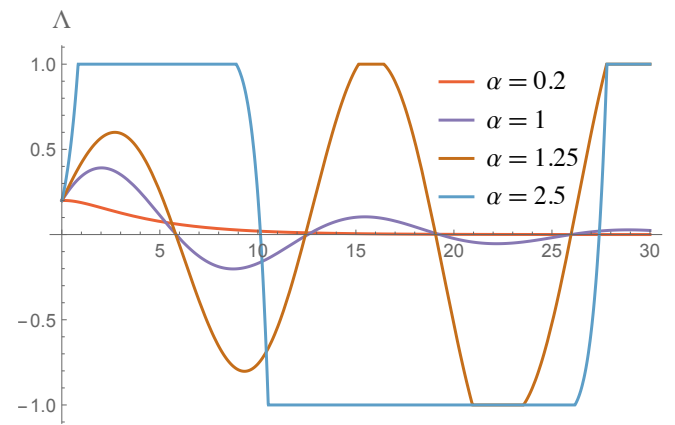

(a) $\eta=0.2$

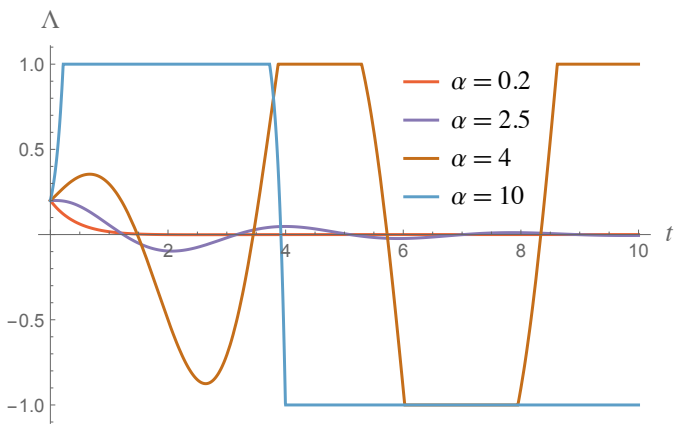

(b) $\eta=2.5$

Figure 3: Numerical solutions of the 2-pin problem 38 computed using the initial condition $H(0)=0.2$, with $h=2$ and various values of $\alpha$ and $\eta$. 


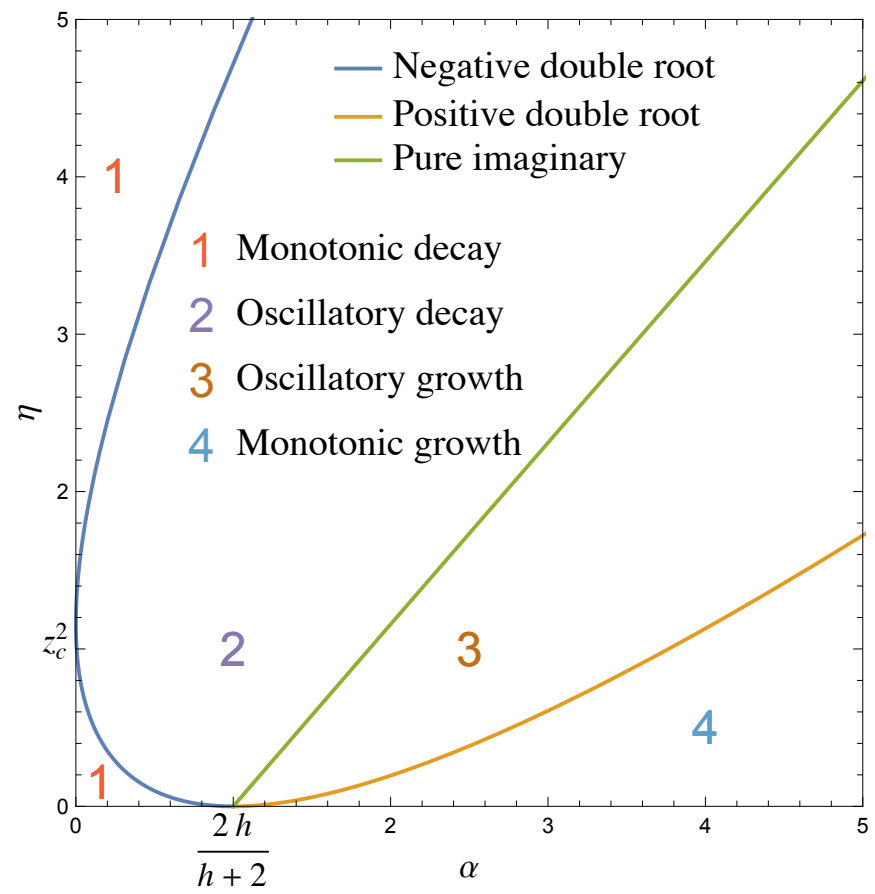

Figure 4: The $(\alpha, \eta)$ parameter space plotted with $h=2$. 


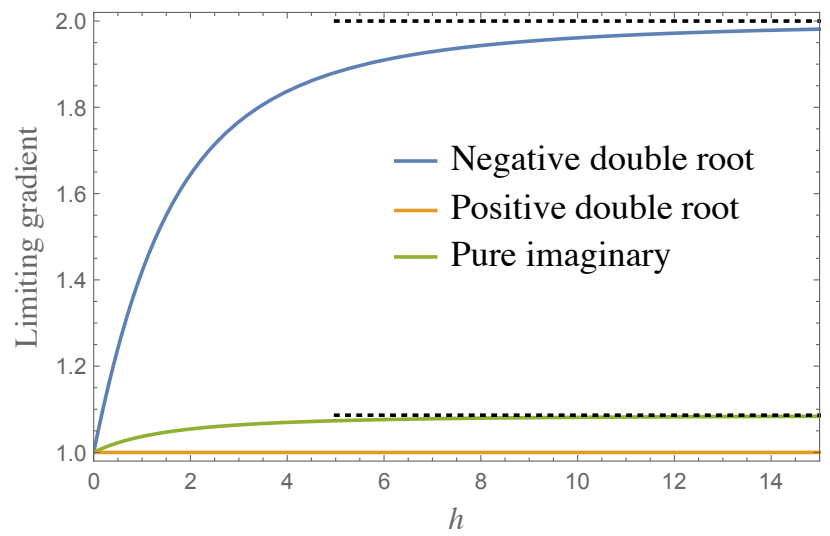

Figure 5: The limiting gradients of the critical curves in the $(\alpha, \eta)$, given by equation (50) (green), 1 (orange) and equation (57) (blue), plotted versus $h$. The dotted lines show the asymptotes $1+\operatorname{sech} \pi$ and 2 approached as $h \rightarrow \infty$. 


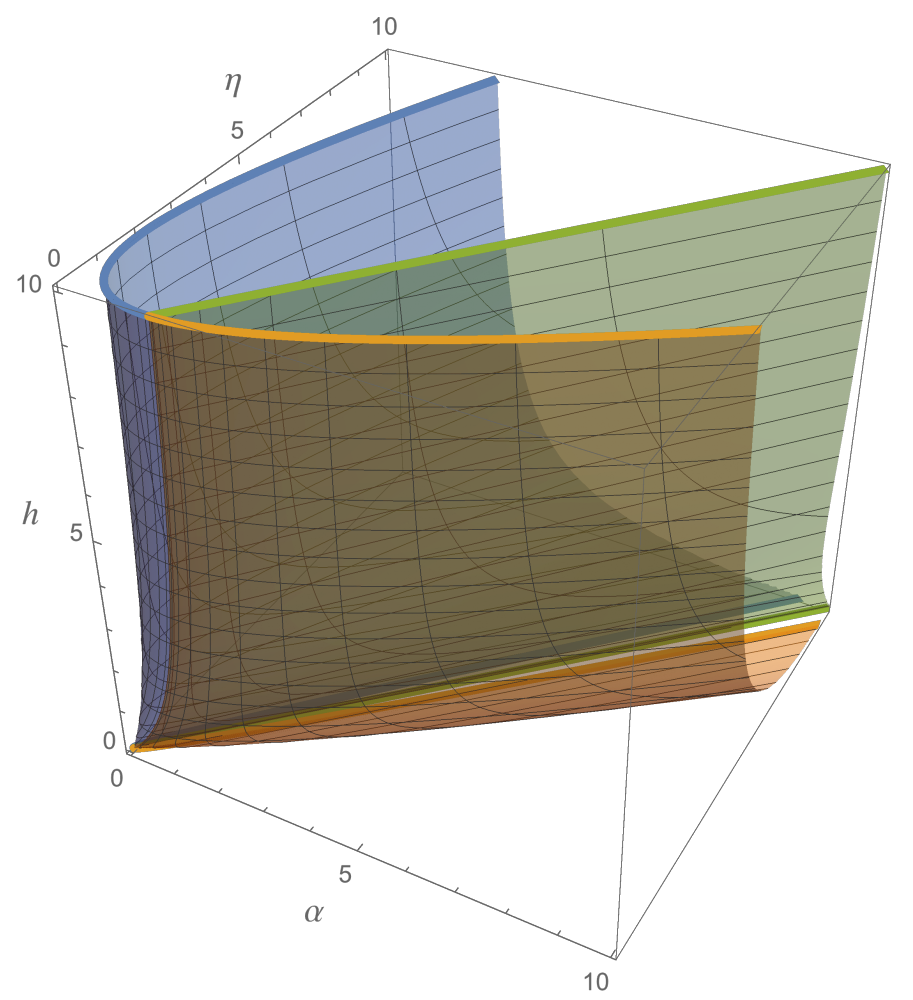

Figure 6: The $(\alpha, \eta, h)$ parameter space. 


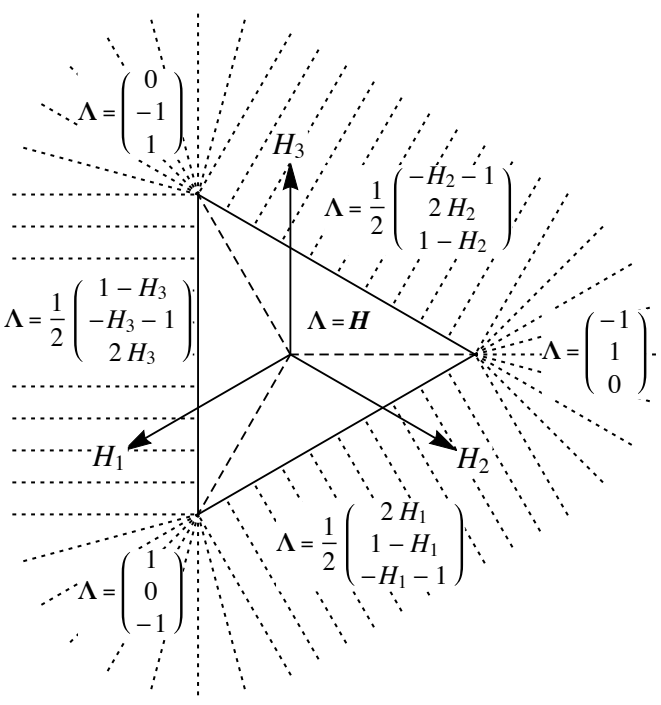

(a)

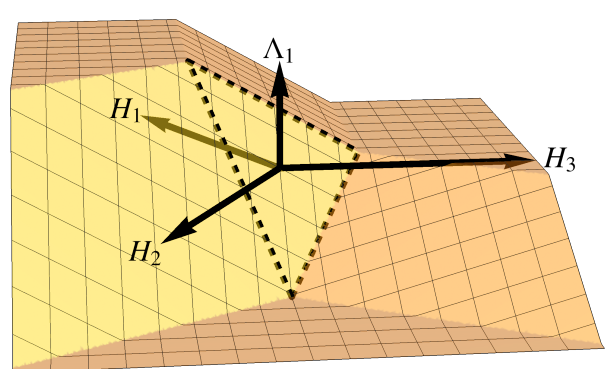

(b)

Figure 7: (a) A sketch of the plane $H_{1}+H_{2}+H_{3}=1$ showing regions where different functional forms of $\boldsymbol{\Lambda}(\boldsymbol{H})$ hold. On the triangle with vertices $\boldsymbol{H}=(0,-1,1),(1,0,-1)$ and $(-1,1,0)$ we have $\boldsymbol{\Lambda}(\boldsymbol{H})=\boldsymbol{H}$; outside the triangle, $\boldsymbol{\Lambda}(\boldsymbol{H})$ is constant on the dashed lines. (b) The first component $\Lambda_{1}$ of $\boldsymbol{\Lambda}$, given by equation $(64)$, plotted versus $\boldsymbol{H}$. The dashed lines mark the image of the equilateral triangle from figure $7(\mathrm{a})$. 


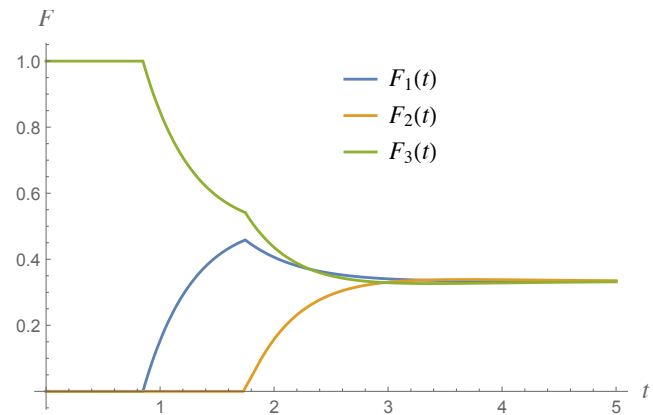

(a) $\alpha=0.2$

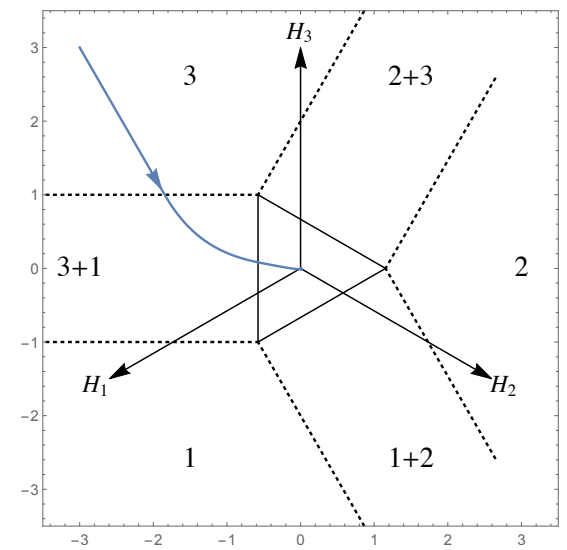

(c) $\alpha=0.2$

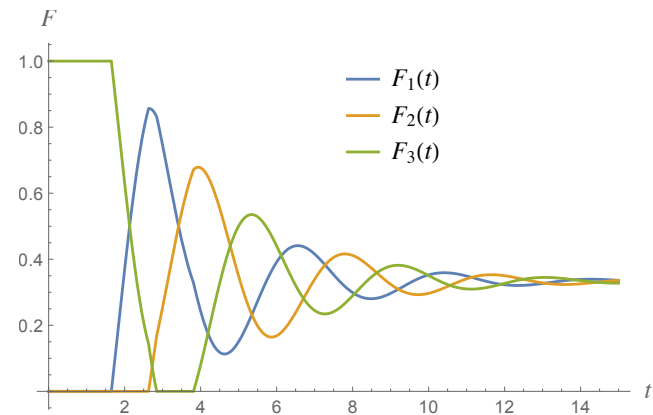

(b) $\alpha=2.5$

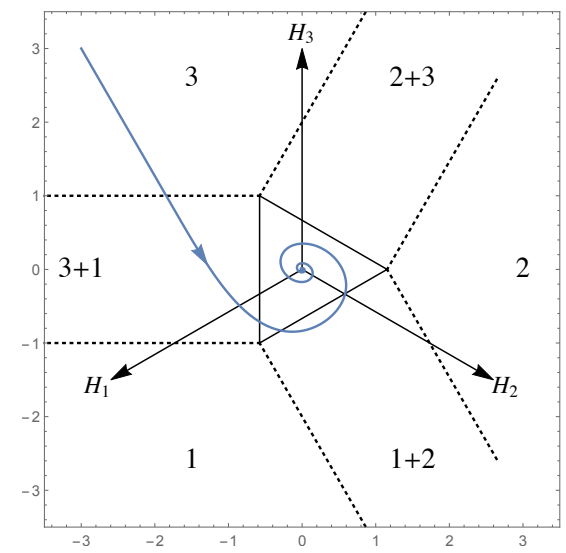

(d) $\alpha=2.5$

Figure 8: Numerical solutions of the 3-pin problem (65) with $h=2, \eta=2.5$ and $\alpha \in\{0.2,2.5\}$. (a) and (b) show the pin forces $\left(F_{1}, F_{2}, F_{3}\right)$ plotted versus time $t$; (c) and (d) show the dynamics plotted in the $\boldsymbol{H}$-plane. 


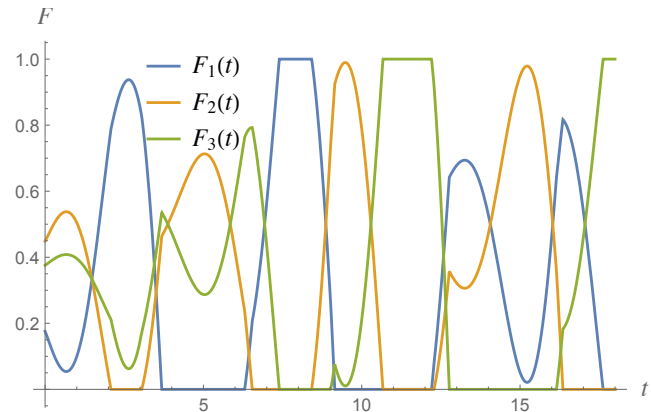

(a) $\alpha=4.0$

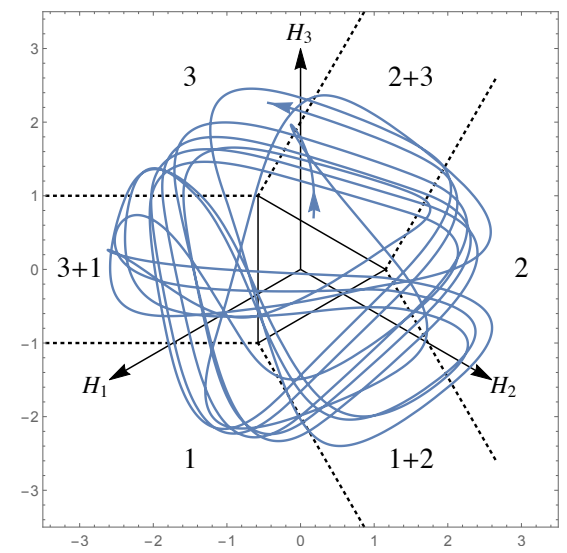

(c) $\alpha=4.0$

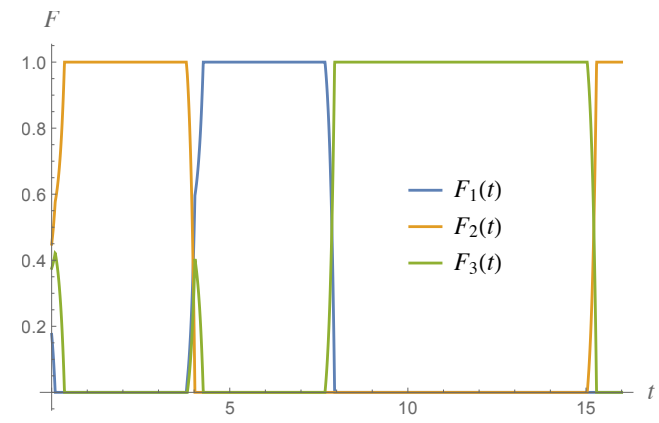

(b) $\alpha=10.0$

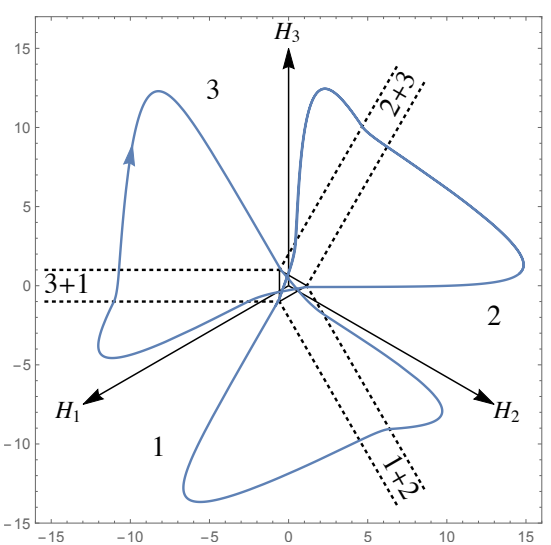

(d) $\alpha=10.0$

Figure 9: Numerical solutions of the 3-pin problem 65 with $h=2, \eta=2.5$ and $\alpha \in\{4.0,10.0\}$. (a) and (b) show the pin forces $\left(F_{1}, F_{2}, F_{3}\right)$ plotted versus time $t$; (c) and (d) show the dynamics plotted in the $\boldsymbol{H}$-plane. 


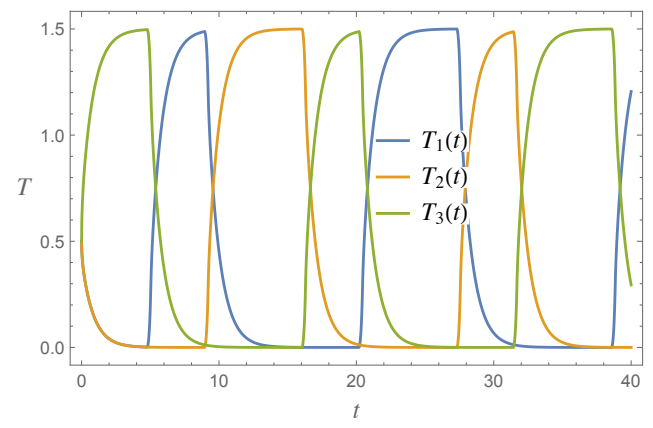

(a)

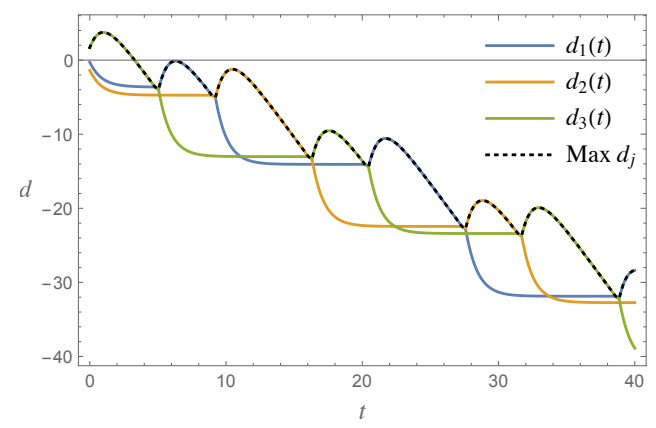

(b)

Figure 10: Numerical solutions of the 3-pin problem equation 65 with $h=2$, $\alpha=10.0$ and $\eta=2.5$. (a) the pin end temperatures $T_{j}(1, t)(j=1,2,3)$ versus time $t$; (b) the pin end displacements $\left(d_{1}, d_{2}, d_{3}\right)$ versus time $t$. 